Relations industrielles

Industrial Relations

\title{
Diary of a Strike. By Professor Bernard Karsh. Urbana: University of Illinois Press, 1958, 180 pp.
}

\section{Jacques Archambault}

Volume 14, numéro 3, juillet 1959

URI : https://id.erudit.org/iderudit/1022297ar

DOI : https://doi.org/10.7202/1022297ar

Aller au sommaire du numéro

Éditeur(s)

Département des relations industrielles de l’Université Laval

ISSN

0034-379X (imprimé)

1703-8138 (numérique)

Découvrir la revue

Citer ce compte rendu

Archambault, J. (1959). Compte rendu de [Diary of a Strike. By Professor Bernard Karsh. Urbana: University of Illinois Press, 1958, 180 pp.] Relations industrielles / Industrial Relations, 14(3), 438-439.

https://doi.org/10.7202/1022297ar

Tous droits réservés @ C Département des relations industrielles de l’Université Laval, 1959
Ce document est protégé par la loi sur le droit d'auteur. L’utilisation des services d'Érudit (y compris la reproduction) est assujettie à sa politique d'utilisation que vous pouvez consulter en ligne.

https://apropos.erudit.org/fr/usagers/politique-dutilisation/ 
En somme, dans ce secteur économique où des forces dynamiques s'affirment, l'arbitrage public devient de plus en plus gênant et ne peut pnétendre qu'à un demi-succès.

Dans le troisième cas étudié, celui des débardeurs, l'expérience de l'arbitrage public et obligatoire s'est soldée par un échec presque complet. Les diffiérences fondamentales des intérêts entre les principales catégories d'employeurs d'une part, et d'autre part l'agressivité des ouvriers ont rendu la vie très dure aux pouvoirs publics. Comme dans l'industrie de la métallungie, les syndicats ouvriers ont résisté vigoureusement à l'ingérence de la cour dans leurs affaires internes. Ils ont souvent préféré l'action directe à l'arbitrage et se sont même souvent révoltés contre les ordres du pouvoir judiciaire. L'auteur étudie assez longuement l'histoire de l'intervention publique dans les conflits au sein de cette industrie, comme il ravait fait dans les deux autres cas, pour plonger le lecteur dans le climat nécessaire à une bonne compréhension du phénomène.

Dans la troisième partie, qui est très courte mais extrêmement importante, l'auteur tente de tirer quelques conclusions. Il insiste sur le fait que le mouvement ouvrier en Australie s'est toujours intéressé autant, sinon plus, à l'action politigue qu'à la négociation collective, ce qui expliquerait partiellement la constance et la vigueur relatives de l'appui qu'il a accordé au système d'arbitrage et aux autres formes d'intervention des pouvoirs publics dans les relations patronales-ouvrières. L'auteur souligne que l'arbitrage a fonctionné passablement bien lorsqu'il a en quelque sorte joué le jeu des parties, mais qu'il a échoué lorsqu'il a voulu s'imposer dans des circonstances qui lui étaient hostiles. Enfin, il met en garde tous les parisans enthousiastes de l'arbitrage public obligatoire contre les mirages du système australien car celui-ci n'a de signification valable que dans le contexte social, économique, culturel et politique du pays où il s'est développé.

La lecture de cet ougrave rendra service à tous ceux qui s'intéressent au règlement des conflits ouvriers et spécialement au problème de l'intervention statutaire des tiers dans les relations patronales-ouvrières. Lorsque l'on discute de changements dans la législation ouvrière, il est souvent question de tribunaux du travail. L'expérience de l'Australie est à cet égard une bonne illustration des avantages et des inconvénients d'un tel système.

Gaston Cholette

Diary of a Strike. By Professor Bernard Karsh. Urbana: University of Illinois Press, 1958, 180 pp.

Voilà un volume fort bien fait et qui mériterait certes une traduction française pour le bénéfice de tous ceux pour lequel une grève apparaît en premier lieu « as a social phenomenon».

D'ailleurs on n'a qu'à voir les têtes de différents chapîtres: Une perspective théorique; Comment cela a-t-il commencé?; Pourquoi se sont-ils organisés en syndicat?; Prélude à la grève; La grève elle-même; Tactiques d'organisation; Pourquoi ont-ils fait la grève?; Analyse des objectifs des travailleurs en cause; Nouvelles relations; Quatre ans après.

La grève dont il est question a eu lieu dans une petite ville américaine de 14,000 habitants située à la tête des Grands-Lacs. Elle a duré quatre mois et a affecté environ 200 travailleurs. Le nom de la ville, des personnes, ont été changés, mais les événements et les persomnes sont réels.

C'est après plusieurs échecs qu'enfin les organisateurs de l'A.F.L. réussirent à organiser l'usine de Taylor. Deux jours après la première réunion publique, Tom Miller, le propriétaine de l'usine adressait une lettre circulaire à chaque travailleur, lettre reproduite. (page 47). Elle commence ainsi: \&It appears that a professional labor Promotor... The Management of this Company does not want a union, because we feel that our employees will not benefit by belonging to the union $\gg$. Just why ambitious outsiders have become to interested in «protecting 》 your rights we do not understand. Perhaps the sizable chunk of money which the union would get from our employees in the form of dues, assessments, etc., is the plum which the union wants to pick ». 
D'ailleurs, les mots « outsiders 》 et « strangers reviennent fréquemment dans le texte.

Ces débuts conduisaient infailliblenents les opposants à un conflit irréductible. Et l'auteur signale que les propriétaires de l'usine jouissaient de la considération de toute la ville. Ils étaient avantageusement reconnus pour leurs oeuvres de charité, leurs dons généreux à l'Eglise Catholique et leur grande influence dans la vie politique et économique de la Cité. Les travailleurs disaient «the Millers have always run this town ».

La partie la plus intéressante se situe au chapitre 7 intitulé \&Why they strike? » L'auteur signale cependant que les interviews sur cette partie ont été faits après la grève «ended in a claimed union victory $\gg, \infty$ qui peut signifier que les opinions auraient pu être toutes autres en une période différente. Deux points particuliers ressortent de ce chapitre.

1) Une fois de plus il s'agissait d'une grève de reconnaissance syndicale.

2) La ligne de piquetage, attaquée, dans les premiers jours par le Maire de la Ville et la police, devint un symbole et prit une signification prépondérante et décisive pour toute la population.

On ne peut évidemment s'empêcher de faire un parallèle entre cette histoire d'une grève et le volume qui a paru, ici au Canada, il y a quelques années, en marge de la grève de l'amiante.

Certes, le volume du professeur Karsh est beaucoup moins considérable, son optique est de toute évidence plus étroite que celui de l'équipe «Cité libre», mais on peut quand même signaler que si quelques sociologues ou chercheurs s'avisaient de faire de chacune de nos grèves de quelque importance. un genre d'étude comme celle du professeur Karsh, notre littérature si pauvre en ce domaine, s'enrichirait d'une véritable enthologie sociale extrêmement utile voire essentielle, car comme le dit l'auteur \& a labor strike is one of the most highy publicised but least studied social phenomena of our time ».

Jacques Archambault
Pourquoi nous travaillons. Jean Fourastié. Coll. : Que sais-je? 》 Presses Universitaires de France, 1959, 126 pp.

Voici un petit ouvrage qui répond à un besoin et qui rendra de grands services. La science économique a pris un essor considérable en ces dernières années, bien qu'elle reste encore loin d'être capable de résoudre tous les problèmes. «Nulle part, plus qu'en économie, l'ignorance n'enveloppe l'humanité ». Cependant qui ne cherche pas à comprendre un peu le monde dans lequel il vit et les foroes qui influencent son destin. Par ailleurs dans les écoles on n'y enseigne à peu près rien. Et il est assez difficile pour un débutant de se lancer dans de profonds traités techniques où il serait facilement perdu. "Le but de ce livre, dit l'auteur, est de mettre à la portée du Français moyen oe qui me paraît être, à l'heure où j'écris, les bases élémentaires d'une science économique concrète, c'est-àdire utile à l'homme d'action et apte à faire mieux comprendre à l'homme de pensée les réalités du monde où il vit. Plus précisément, ce livre s'adresse aux autodidactes ».

Même si les données de bases d'où part l'auteur pour illustrer ses exposés sont tirées de la réalité française, cet ouvrage peut être très utile aux Canadiens, car ils pourront facilement effectuer les transpositions nécessaires. Après une introduction où l'auteur nous fait passer de la réalité quotidienne à la science économique, six chapitres viennent nous présenter les principaux problèmes de l'économique: Pourquoi nous travaillons; Comment nous travaillons; Le rationnement et les revenus; Les revenus et les prix; L'emploi et les salaires; Le salaire indirect; La sécurité sociale; Niveau de vie, genre de vie.

G.D.

L'Action sociale des catholiques en France (1871-1914), par Henri Rollet, tome second. Desclée de Brouwer, Paris, 1958, 404 pp.

Dans ce tome second l'auteur, un chercheur consciencieux, a relevé et raconté tout ce que les catholiques de France ont fait ou tenté de faire dans 